\title{
GENERATING FUNCTIONS FOR JACOBI AND \\ LAGUERRE POLYNOMIALS
}

H. M. SRIVASTAVA ${ }^{1}$

Let $v$ be a function of $t$ defined by

$$
v=t(1+v)^{b+1}, \quad v(0)=0 .
$$

Then it follows from Lagrange's expansion formula [6, Vol. I, p. 126, Ex. 212] that

$$
\frac{(1+v)^{a+1}}{1-b v}=\sum_{n=0}^{\infty}\left(\begin{array}{c}
a+(b+1) n \\
n
\end{array}\right) t^{n} .
$$

Making use of the formula (2), Carlitz [2] has proved that the Laguerre polynomial $L_{n}^{(a+b n)}(x)$, where

$$
L_{n}^{(\alpha)}(x)=\sum_{k=0}^{n}(-1)^{k}\left(\begin{array}{l}
\alpha+n \\
n-k
\end{array}\right) \frac{x^{k}}{k !}
$$

satisfies a generating relation in the form

$$
\sum_{n=0}^{\infty} L_{n}^{(a+b n)}(x) t^{n}=\frac{(1+v)^{a+1}}{1-b v} \exp (-x v)
$$

where $v$ is given by (1) and $a, b$ are arbitrary complex numbers. Note that the special case of (4) when $b$ is an arbitrary integer was proved earlier by Brown [1].

In terms of the generalized hypergeometric function

$$
{ }_{p} F_{q}\left[\begin{array}{c}
\alpha_{1}, \cdots, \alpha_{p} ; \\
\beta_{1}, \cdots, \beta_{q} ;
\end{array}\right]=\sum_{n=0}^{\infty} \frac{\prod_{j=1}^{p}\left(\alpha_{j}\right)_{n}}{\prod_{j=1}^{q}\left(\beta_{j}\right)_{n}} \frac{x^{n}}{n !},
$$

where

$$
(\lambda)_{n}=\lambda(\lambda+1)(\lambda+2) \cdots(\lambda+n-1), \quad n \geqq 1,(\lambda)_{0}=1,
$$

the generating relation (4) assumes the form

Received by the editors March 22, 1969.

1 Present address. Department of Mathematics, University of Victoria, Victoria, British Columbia, Canada. 


$$
\begin{aligned}
\sum_{n=0}^{\infty}\left(\begin{array}{c}
a+(b+1) n \\
n
\end{array}\right){ }_{1} F_{1}\left[\begin{array}{r}
-n ; \\
x \\
1+a+b n ;
\end{array}\right] t^{n} & \\
& =\frac{(1+v)^{a+1}}{1-b v} \exp (-x v) .
\end{aligned}
$$

In (7) if we replace $x$ by $x z$, multiply both sides by $z^{\lambda-1}$ and take their Laplace transforms with respect to the variable $z$, we shall readily obtain

$$
\begin{aligned}
\sum_{n=0}^{\infty}\left(\begin{array}{c}
a+(b+1) n \\
n
\end{array}\right){ }_{2} F_{1}\left[\begin{array}{r}
-n, \lambda ; \\
1+a+b n ;
\end{array}\right] t^{n} & \\
& =\frac{(1+v)^{a+1}}{1-b v}(1+x v)^{-\lambda}
\end{aligned}
$$

where the binomial $(1+x v)^{-\lambda}$ may be written as an ${ }_{1} F_{0}$.

The form of (8) suggests the existence of the general formula

$$
\sum_{n=0}^{\infty}\left(\begin{array}{c}
a+(b+1) n \\
n
\end{array}\right)_{p+1} F_{q+1}\left[\begin{array}{r}
-n, \alpha_{1}, \cdots, \alpha_{p} ; \\
x \\
1+a+b n, \beta_{1}, \cdots, \beta_{q} ;
\end{array}\right] t^{n}
$$

$$
=\frac{(1+v)^{a+1}}{1-b v}{ }_{p} F_{q}\left[\begin{array}{l}
\alpha_{1}, \cdots, \alpha_{p} ; \\
\beta_{1}, \cdots, \beta_{q} ;
\end{array}\right] \text {, }
$$

where $p, q$ are nonnegative integers, the $\alpha$ 's and $a, b$ take general values, real or complex, and

$$
\beta_{j} \neq 0,-1,-2, \cdots, \quad j=1,2, \cdots, q .
$$

The derivation of (9) from (7) and (8) by the principle of multidimensional mathematical induction would require the Laplace and inverse Laplace transform techniques illustrated, for instance, by the author [7].

For a direct proof without using (7) and (8) we notice that, in view of the definition (5), 


$$
\begin{aligned}
\sum_{n=0}^{\infty}\left(\begin{array}{c}
a \\
+(b+1) n \\
n
\end{array}\right){ }_{p+1} F_{a+1}\left[\begin{array}{c}
-n, \alpha_{1}, \cdots, \alpha_{p} ; \\
1+a+b n, \beta_{1}, \cdots, \beta_{q} ;
\end{array}\right] t^{n} \\
=\sum_{n=0}^{\infty} t^{n} \sum_{k=0}^{n}(-1)^{k}\left(\begin{array}{c}
a+(b+1) n \\
n-k
\end{array}\right) \frac{\prod_{j=1}^{p}\left(\alpha_{j}\right)_{k}}{\prod_{j=1}^{q}\left(\beta_{j}\right)_{k}} \frac{x^{k}}{k !} \\
=\sum_{k=0}^{\infty}(-1)^{k} \frac{\prod_{j=1}^{p}\left(\alpha_{j}\right)_{k}}{\prod_{j=1}^{q}\left(\beta_{j}\right)_{k}} \frac{x^{k}}{k !} \sum_{n=k}^{\infty}\left(\begin{array}{c}
a+(b+1) n \\
n-k
\end{array}\right) t^{n} \\
=\sum_{k=0}^{\infty}(-1)^{k} \frac{\prod_{j=1}^{p}\left(\alpha_{j}\right)_{k}}{\prod_{j=1}^{q}\left(\beta_{j}\right)_{k}} \frac{x^{k} t^{k}}{k !} \sum_{n=i}^{\infty}\left(\begin{array}{c}
a+(b+1) k+(b+1) n \\
k
\end{array}\right) t^{n} \\
=\sum_{k=0}^{\infty}(-1)^{k} \frac{\prod_{j=1}^{p}\left(\alpha_{j}\right)_{k}}{\prod_{j=1}^{q}\left(\beta_{j}\right)_{k}} \frac{x^{k} t^{k}}{k !} \frac{(1+v)^{a+(b+1) k+1}}{1-b v}
\end{aligned}
$$

by (2), and the formula (9) follows immediateiy. We can easily attribute a direct proof to the formula (8) which obviously corresponds to the special case $p=1, q=0$ of (9).

A similar generalization of Carlitz's formula [2, p. 827, Equation (16)] has the form

$$
\begin{gathered}
\sum_{n=0}^{\infty}\left(\begin{array}{c}
-a-b n \\
n
\end{array}\right){ }_{p+1} F_{q+1}\left[\begin{array}{r}
-n, \alpha_{1}, \cdots, \alpha_{p} ; \\
1-a-(b+1) n, \beta_{1}, \cdots, \beta_{q} ;
\end{array}\right] t^{n} \\
=\frac{A(-t, a, b)}{1-B(-t, b)}{ }_{p} F_{q}\left[\begin{array}{r}
\alpha_{1}, \cdots, \alpha_{p} ; \\
-\frac{x B(-t, b)}{1-B(-t, b)} \\
\beta_{1}, \cdots, \beta_{q} ;
\end{array}\right],
\end{gathered}
$$

where, for convenience,

$$
B(t, b)=-\sum_{n=1}^{\infty}\left(\begin{array}{c}
(b+1) n \\
n-1
\end{array}\right) \frac{t^{n}}{n}
$$

and

$$
A(t, a, b)=\frac{[1-B(t, b)]^{a+1}}{1+b B(t, b)} .
$$

Indeed the formula (11) is obtainable from (9) by replacing $a$ by $-a$ and $b$ by $-(b+1)$. 
It may be of interest to remark that for $b=0$ and $b=-1$ the formula (9) yields Chaundy's results (25) and (27) respectively (see $\left[4\right.$, p. 62]). For $b=-\frac{1}{2}$, (9) reduces to the generating relation (7), p. 264 of Brown's recent paper. ${ }^{2}$

For the Jacobi polynomial defined by

$$
P_{n}^{(\alpha, \beta)}(x)=\sum_{k=0}^{n}\left(\begin{array}{c}
\alpha+n \\
k
\end{array}\right)\left(\begin{array}{c}
\beta+n \\
n-k
\end{array}\right)\left(\frac{x-1}{2}\right)^{n-k}\left(\frac{x+1}{2}\right)^{k},
$$

it is easy to show from the identity $(4.22 .1)$ of $[8, \mathrm{p} .63]$ that

$$
\left.P_{n}^{(\alpha-n, \beta-n)}(x) \text { ( } \begin{array}{c}
n-\alpha-\beta-1 \\
n
\end{array}\right)\left(\begin{array}{c}
1-x \\
2
\end{array}\right)^{n}{ }_{2} F_{1}\left[\begin{array}{c}
-n,-\alpha ; \\
\frac{2}{1-x} \\
-\alpha-\beta ;
\end{array}\right],
$$

and therefore (8) gives us the elegant generating function

(16) $\sum_{n=0}^{\infty} P_{n}^{(\alpha-n, \beta-(b+1) n)}(x) t^{n}=(1+w)^{-\alpha-\beta}(1+b w)^{-1}\left(1+\frac{2 w}{1-x}\right)^{\alpha}$,

where

$$
w=\frac{1}{2}(1-x) t(1+w)^{b+1} .
$$

Evidently (16) reduces to the known formula [3, p. 88]

$$
\sum_{n=0}^{\infty} P_{n}^{(\alpha-n, \beta-n)}(x) t^{n}=\left[1+\frac{1}{2}(x+1) t\right]^{\alpha}\left[1+\frac{1}{2}(x-1) t\right]^{\beta}
$$

when $b=0$, and for $b=-1$ it leads us to Feldheim's result $[5$, p.120 $]$

$$
\sum_{n=0}^{\infty} P_{n}^{(\alpha-n, \beta)}(x) t^{n}=(1+t)^{\alpha}\left[1-\frac{1}{2}(x-1) t\right]^{-\alpha-\beta-1} .
$$

Now from the definition (14) we readily have $[8$, p. 61]

$$
P_{n}^{(\alpha, \beta)}(x)=\left(\begin{array}{c}
\alpha+n \\
n
\end{array}\right){ }_{2} F_{1}\left[\begin{array}{r}
-n, 1+\alpha+\beta+n ; \\
\frac{1-x}{2} \\
1+\alpha ;
\end{array}\right],
$$

whence it follows at once that

$2 \mathrm{~J}$. W. Brown, New generating functions for classical polynomials, Proc. Amer. Math. Soc. 20 (1969), 263-268. 


$$
P_{n}^{(\alpha+b n, \beta-(b+1) n)}(x)=\left(\begin{array}{c}
\alpha+(b+1) n \\
n
\end{array}\right){ }_{2} F_{1}\left[\begin{array}{r}
-n, 1+\alpha+\beta \\
\frac{1-x}{2} \\
1+\alpha+b n
\end{array}\right]
$$

Consequently, (8) gives us another class of generating functions for the Jacobi polynomial in the form

$$
\begin{aligned}
\sum_{n=0}^{\infty} P_{n}^{(\alpha+b n, \beta-(b+1) n)}(x) t^{n} & \\
& =(1+v)^{\alpha+1}(1-b v)^{-1}\left[1-\frac{1}{2}(x-1) v\right]^{-\alpha-\beta-1},
\end{aligned}
$$

where $v$ is defined by (1) and $b, \alpha, \beta$ are unrestricted, in general.

For $b=-1,(22)$ leads us again to Feldheim's formula (19); when $b=0$, it reduces to the generating relation

$$
\sum_{n=0}^{\infty} P_{n}^{(\alpha, \beta-n)}(x) t^{n}=(1-t)^{\beta}\left[1-\frac{1}{2}(x+1) t\right]^{-\alpha-\beta-1}
$$

also due to Feldheim [5, p. 120].

Finally, we remark that the special case $b=-\frac{1}{2}$ of our formula (22) corresponds to

$$
\begin{aligned}
& \sum_{n=0}^{\infty} P_{n}^{(\alpha-n / 2, \beta-n / 2)}(x) t^{n} \\
&=[1+u(t)]^{\alpha+1}\left[1+\frac{1}{2} u(t)\right]^{-1}\left[1-\frac{1}{2}(x-1) u(t)\right]^{-\alpha-\beta-1},
\end{aligned}
$$

where

$$
u(t)=\frac{1}{2} t\left[t+\sqrt{ }\left(t^{2}+4\right)\right] .
$$

The formula (24) appears in Brown's recent paper referred to earlier.

ADDED IN PROOF. In a private communication to the author, Professor L. Carlitz suggests that following the method of proof of the formula (9) one can readily obtain its straightforward generalization in the form

$$
\sum_{n=0}^{\infty}\left(\begin{array}{c}
a+(b+1) n \\
n
\end{array}\right) t^{n} \sum_{k=0}^{n} \frac{(-n)_{k} c_{k}}{(1+a+b n)_{k}} \frac{x^{k}}{k !}
$$

$$
=\frac{(1+v)^{a+1}}{1-b v} \sum_{k=0}^{\infty} c_{k} \frac{(-x v)^{k}}{k !},
$$


where the $c_{k}$ are arbitrary constants and $v$ is defined by (1). It seems worthwhile to remark here that further extensions of $(*)$ form the subject-matter of our discussion in a forthcoming paper.

\section{REFERENCES}

1. J. W. Brown, On zero type sets of Laguerre polynomials, Duke Math. J. 35 (1968), 821-823.

2. L. Carlitz, Some generating functions for Laguerre polynomials, Duke Math. J. 35 (1968), 825-827.

3. L. Carlitz, $A$ bilinear generating function for the Jacobi polynomials, Boll. Un. Mat. Ital. (3) 18 (1963), 87-89.

4. T. W. Chaundy, An extension of hypergeometric functions, Quart. J. Math. Oxford Ser. 14 (1943), 55-78.

5. E. Feldheim, Relations entre les polynomes de Jacobi, Laguerre et Hermite, Acta Math. 74 (1941), 117-138.

6. G. Pólya and G. Szegö, A ufgaben und Lehrsätze aus der Analysis, Springer-Verlag, Berlin, 1925.

7. H. M. Srivastava, An extension of the Hille-Hardy formula, Math. Comp. 23 (1969), 305-311; see also Notices Amer. Math. Soc. 15 (1968), 634-635.

8. G. Szegö, Orthogonal polynomials, Amer. Math. Soc. Colloq. Publ., vol. 23, Amer. Math. Soc., Providence, R. I., 1939.

West Virginia UNiversity 